\title{
The Value of Adding Red Cell Distribution Width to Mehran Risk Score to Predict Contrast-induced Acute Kidney Injury in Patients with Acute Coronary Syndrome
}

Sherif Elhosseiny ${ }^{1}$, Tamer Akel ${ }^{1}$, $\operatorname{Jad}_{\text {Mroue }}{ }^{1}$, Praveena Tathineni ${ }^{2}$, Suzanne El Sayegh ${ }^{3}$, James Lafferty ${ }^{4}$

1. Internal Medicine, Staten Island University Hospital, Staten Island, USA 2. Internal Medicine, Touro, Staten Island, USA 3. Nephrology, Staten Island University Hospital, Staten Island, USA 4. Cardiology, Staten Island University Hospital, Staten Island, USA

$\square$ Corresponding author: Sherif Elhosseiny, selhossein@northwell.edu Disclosures can be found in Additional Information at the end of the article

\section{Abstract}

Background: Contrast-induced acute kidney injury (CI-AKI) is a relatively reversible cause of acute kidney injury (AKI) that occurs after radiocontrast media administration. It is a common complication after percutaneous coronary intervention, especially in patients with acute coronary syndrome (ACS). The aim of this study is to determine the utility of red cell distribution width (RDW) in predicting CI-AKI in patients with ACS and to determine the value of adding RDW to the Mehran risk score (MRS) on admission.

Methods: A total of 161 patients who presented with ST-elevation myocardial infarction (STEMI) or non-STEMI were identified retrospectively between January 2015 and December 2016. Patients were divided into two groups, those who developed CI-AKI after percutaneous coronary intervention (PCI) and those who did not.

Results: A total of 161 patients were analyzed. Of them, 12 developed CI-AKI (eight presented with STEMI and four presented with non-STEMI). RDW did not correlate with the development of CI-AKI $(14.55 \pm 1.48$ vs $14.83 \pm 1.21 ; \mathrm{p}=0.072)$. The areas under the receiver operating characteristic curves (ROCs) for RDW, MRS, and the combined model (MRS and RDW) for the prediction of CI-AKI were 0.721 (95\% confidence interval (CI), 0.645 to 0.788 ; $\mathrm{p}=0.0024$ ), 0.885 ( $95 \%$ CI, 0.825 to 0.930 ; $\mathrm{p}<0.0001$ ), 0.890 (95\% CI, 0.831 to 0.933 ; $\mathrm{p}<0.0001$ ), respectively. Pairwise comparisons between ROCs for MRS vs the combined model yielded a non-significant p-value of 0.49. This signifies no added benefit for RDW to MRS for predicting CI-AKI.

Received 06/08/2018 Review began 06/18/2018 Review ended 06/27/2018 Published 07/02/2018

\section{C) Copyright 2018}

Elhosseiny et al. This is an open access article distributed under the terms of the Creative Commons Attribution License CC-BY 3.0., which permits unrestricted use, distribution, and reproduction in any medium, provided the original author and source are credited.
Conclusion: RDW does not correlate with the development of CI-AKI in patients with ACS. The Mehran risk score remains a better indicator of CI-AKI risk assessment with no role for the addition of RDW to it. Further studies are needed to better assess predictors of CI-AKI in patients undergoing percutaneous coronary intervention.

Categories: Cardiology, Internal Medicine, Nephrology

Keywords: acute coronary syndrome, red blood cell distribution width, contrast induced kidney injury

\section{Introduction}

How to cite this article

Elhosseiny S, Akel T, Mroue J, et al. (July 02, 2018) The Value of Adding Red Cell Distribution Width to Mehran Risk Score to Predict Contrast-induced Acute Kidney Injury in Patients with Acute Coronary Syndrome . Cureus 10(7): e2911. DOI 10.7759/cureus.2911 
Contrast-induced acute kidney injury (CI-AKI) is a well-recognized complication observed after the administration of iodinated contrast media during angiographic procedures [1]. It is defined as a greater than $25 \%$ increase in baseline serum creatinine or an absolute increase of $0.5 \mathrm{mg} / \mathrm{dL}$ within 48 hours after exposure and usually peaking within the following five days [1]. CI-AKI has been associated with adverse outcomes, including the need for renal replacement therapy, in-hospital complications, rehospitalization, increased length of stay, and mortality [2-3]. The need for contrast-based diagnostic and therapeutic cardiovascular procedures has been constantly increasing over the past decade. Furthermore, patients with acute coronary syndrome (ACS) have been observed to have nearly double the rates of CI-AKI [4-5]. Therefore, all patients undergoing percutaneous coronary angiography (PCI) should be evaluated for CIAKI risk. At present, several risk scores have been developed. Of them, the Mehran risk score (MRS) remains the most widely used risk assessor for predicting CI-AKI risk [6]. In this retrospective study, we evaluated the predictive utility of red cell distribution width (RDW) for CI-AKI solely and when added to the Mehran risk score.

\section{Materials And Methods}

This is a single centered, retrospective cohort study done at a major community hospital in New York City and in one of the most diverse communities in the United States. Patients who presented to our hospital between January 2015 and December 2016 with a diagnosis of acute coronary syndrome (ACS) were identified. Only patients with ST-elevation myocardial infarction (STEMI) or non-STEMI who underwent PCI were included. A total of 161 patients were included and were divided into those who developed CI-AKI and those who didn't. CI-AKI was defined as a greater than $25 \%$ increase in baseline serum creatinine or an absolute increase of $0.5 \mathrm{mg} / \mathrm{dL}$ within 48 hours after receiving contrast media for PCI. Patients with end-stage renal disease on hemodialysis, liver cirrhosis, autoimmune disease, active infection, and hematological malignancies were excluded. Demographics and laboratory characteristics were identified and analyzed on admission in both groups.

For continuous variables, the differences between the groups were estimated using the Wilcoxon two-sample test. For categorical variables, Fisher's exact test was used. All statistical tests were two-sided and conducted at a 0.05 level of significance. A logistic regression analysis was performed to assess the predictive value of RDW. A receiver operating characteristic (ROC) curve analysis was performed to identify the optimal cut-off point for RDW, Mehran risk score, and the model at which sensitivity and specificity would be maximal for the prediction of CIAKI. Areas under the curve (AUC) were calculated and a pairwise comparison of ROC curves was done.

\section{Results}

Of the 161 patients identified and analyzed, 149 didn't have CI-AKI while 12 did (eight presented with STEMI and four presented with non-STEMI). Baseline characteristics for both groups are presented in Table 1. RDW didn't correlate with the development of CI-AKI (14.55 \pm 1.48 vs $14.83 \pm 1.21 ; \mathrm{p}=0.072$ ). MRS was predictive of CI-AKI in the included subjects. Congestive heart failure (CHF), diabetes mellitus (DM), use of an intra-aortic balloon pump (IABP), hemoglobin level (Hb), blood urea nitrogen (BUN), and the estimated glomerular filtration rate (GFR) were all significant predictors of CI-AKI and are all components of the Mehran risk score except blood urea nitrogen (BUN), as presented in Table 2. The logistic regression analysis failed to show any predictive value for RDW in CI-AKI (Table 3). The areas under the receiver operating characteristic curves (ROCs) for RDW, MRS, and the combined model (MRS and RDW) for the prediction of CI-AKI were 0.721 (95\% confidence interval (CI), 0.645 to $0.788 ; \mathrm{p}=0.0024$ ), 0.885 (95\% CI, 0.825 to $0.930 ; \mathrm{p}<0.0001$ ), and 0.890 (95\% CI, 0.831 to $0.933 ; \mathrm{p}<0.0001)$, respectively. A pairwise comparison of ROC curves failed to show any additional value for RDW to the Mehran risk score ( $\mathrm{p}=0.49)$ (Figure 1). 


\section{Cureus}

\begin{tabular}{|c|c|c|c|}
\hline & No CI-AKI (149) & CI-AKI (12) & p-Value \\
\hline Age (years) & $62.5 \pm 11$ & $66.2 \pm 11$ & $0.26^{b}$ \\
\hline Male gender, $\mathrm{n}(\%)$ & $109(73)$ & $8(66)$ & $0.74^{\mathrm{a}}$ \\
\hline Height (M) & $1.7 \pm 0.1$ & $1.68 \pm 0.06$ & $0.39^{b}$ \\
\hline Weight (Kg) & $86.8 \pm 17.7$ & $85 \pm 10.6$ & $0.84^{b}$ \\
\hline BMI & $29.88 \pm 5.30$ & $30.21 \pm 4.61$ & $0.77^{\mathrm{b}}$ \\
\hline STEMI, n (\%) & $72(48)$ & $8(67)$ & $0.37^{\mathrm{a}}$ \\
\hline Non-STEMI, n (\%) & $76(51)$ & $4(33)$ & $0.37^{\mathrm{a}}$ \\
\hline Hypertension, n (\%) & $103(69)$ & $11(92)$ & $0.18^{\mathrm{a}}$ \\
\hline Dyslipidemia, n (\%) & $92(62)$ & $9(75)$ & $0.54^{\mathrm{a}}$ \\
\hline COPD, n (\%) & $2(1)$ & $0(0)$ & $1.00^{\mathrm{a}}$ \\
\hline Prior PCl, n (\%) & $26(17)$ & $4(33)$ & $0.24^{\mathrm{a}}$ \\
\hline Congestive heart failure, $\mathrm{n}(\%)$ & $10(6)$ & $4(33)$ & $0.01^{\mathrm{a}}$ \\
\hline Diabetes mellitus, $n$ (\%) & $31(21)$ & $7(58)$ & $0.01^{\mathrm{a}}$ \\
\hline No PMHx, n (\%) & $26(17)$ & $1(8)$ & $0.69^{\mathrm{a}}$ \\
\hline IABP use, $n(\%)$ & $1(1)$ & $2(16)$ & $0.01^{\mathrm{a}}$ \\
\hline Amount of used contrast (ml) & $142.72 \pm 66.49$ & $108.75 \pm 112.94$ & $0.11^{b}$ \\
\hline Hospital stay length (days) & $5 \pm 4$ & $12 \pm 18$ & $0.24^{b}$ \\
\hline In-hospital expiration, n (\%) & $2(1)$ & $5(42)$ & $<.0001^{\mathrm{a}}$ \\
\hline \multicolumn{4}{|c|}{$\begin{array}{l}\text { Abbreviations: a: Fisher's exact test; b: Wilcoxon two-sample test; BMI: body mass index; CI-AKI: contrast-induced acute kidne } \\
\text { injury; COPD: chronic obstructive pulmonary disease; IABP: intra-aortic balloon pump; PCI: percutaneous coronary intervention } \\
\text { PMHX: past medical history; STEMI: ST-elevation myocardial infarction }\end{array}$} \\
\hline
\end{tabular}




\section{Cureus}

\begin{tabular}{|c|c|c|c|}
\hline & No CI-AKI (149) & CI-AKI (12) & p-value \\
\hline Hemoglobin (g/dl) & $14.13 \pm 1.78$ & $12.75 \pm 2.49$ & $0.01^{\mathrm{b}}$ \\
\hline RDW (\%) & $14.035 \pm 1.48$ & $14.83 \pm 1.21$ & $0.07^{b}$ \\
\hline MCV (FL) & $87.33 \pm 6.7$ & $84.83 \pm 5.28$ & $0.18^{\mathrm{b}}$ \\
\hline Neutrophils count $\left(10^{3} / \mu \mathrm{L}\right)$ & $6.50 \pm 3.23$ & $6.93 \pm 2.64$ & $0.70^{\mathrm{b}}$ \\
\hline Platelets count $\left(10^{3} / \mu \mathrm{L}\right)$ & $241.22 \pm 92.30$ & $218.00 \pm 79.76$ & $0.40^{\mathrm{b}}$ \\
\hline Lymphocytes count $\left(10^{9} / \mathrm{L}\right)$ & $2.67 \pm 2.69$ & $2.30 \pm 1.03$ & $0.64^{b}$ \\
\hline $\mathrm{N} / \mathrm{L}$ ratio & $3.64 \pm 4.10$ & $2.95 \pm 2.95$ & $0.97^{\mathrm{b}}$ \\
\hline $\mathrm{P} / \mathrm{L}$ ratio & $122.37 \pm 73.26$ & $111.29 \pm 53.26$ & $0.61^{\mathrm{b}}$ \\
\hline BUN (mg/dl) & $18.48 \pm 9.64$ & $29.58 \pm 13.34$ & $<0.001^{b}$ \\
\hline Creatinine (mg/dl) & $1.08 \pm 0.41$ & $1.96 \pm 0.61$ & $<.0001^{\mathrm{b}}$ \\
\hline EGFR $(\mathrm{ml} / \mathrm{min} / 1.73 \mathrm{~m})$ & $72.42 \pm 21.45$ & $35.75 \pm 16.91$ & $<.0001^{\mathrm{b}}$ \\
\hline Creatinine within 48 hours (mg/dl) & $1.02 \pm 0.34$ & $2.93 \pm 0.82$ & $<.0001^{\mathrm{b}}$ \\
\hline RDW (\%) & $14.55 \pm 1.48$ & $14.83 \pm 1.21$ & $0.07^{\mathrm{b}}$ \\
\hline Mehran risk score & $4.60 \pm 3.51$ & $11.33 \pm 4.58$ & $<.0001^{b}$ \\
\hline
\end{tabular}

\section{TABLE 2: Laboratory parameters of the included patients}

Abbreviations: a: Fisher's exact test; b: Wilcoxon two-sample test; BUN: blood urea nitrogen; CI-AKI: contrast-induced acute kidney injury; eGFR: estimated glomerular filtration rate; MCV: mean corpuscular volume; N/L: neutrophil/lymphocyte; P/L: platelets/lymphocytes; RDW: red blood cell distribution width

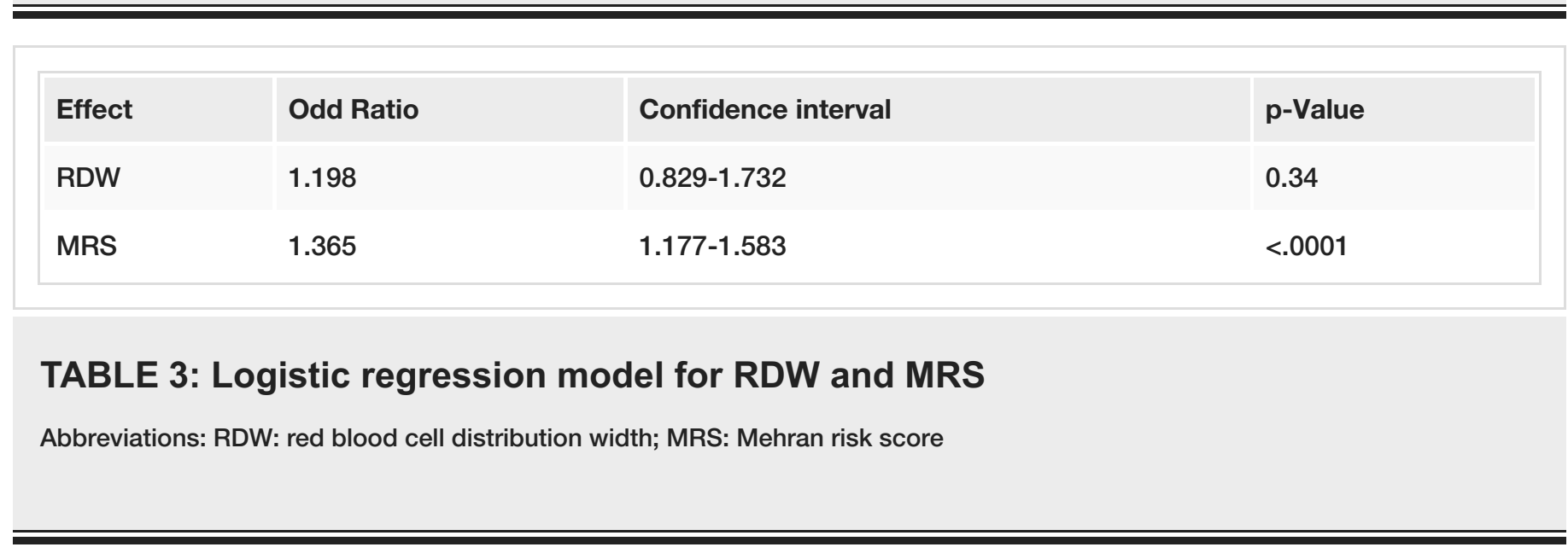




\section{Cureus}



FIGURE 1: ROCs for RDW, MRS, and model with pairwise comparison between MRS and model giving a significance value $(p=0.49)$

ROC: receiver operating characteristic; MRS: Mehran risk score

\section{Discussion}

Each year, more than 80-million iodinated contrast studies are performed across the globe. The trend towards minimally invasive diagnostic and interventional procedures that require the use of intravenous or intra-arterial contrast has been increasing [7]. With this increase, the incidence of CI-AKI has also been rising. In fact, cohort data have shown that CI-AKI is the third most common cause of AKI in patients admitted to the hospital [8]. Nevertheless, the exact pathophysiology of CI-AKI remains obscure, and several inflammatory mediators may play a role in oxidative stress and apoptosis.

In this study, we evaluated the role of red blood cell distribution width (RDW) as a predictive marker for CI-AKI. RDW is a numerical measure of red blood cell (RBC) volume variations. It is part of a standard complete blood count and is useful in eliciting the differential diagnosis of anemia. It has been associated with mortality in coronary artery disease (CAD) patients along with a higher rate of major cardiovascular adverse events [9-10]. The role of RDW in predicting contrast-induced acute kidney injury (CI-AKI) has been investigated previously. Mizuno et al. demonstrated that RDW was independently associated with CI-AKI and has an additional predictive value to the MRS [11]. Akkoyun et al. and Akin et al. also showed that RDW is an independent predictor for CI-AKI in STEMI patients undergoing PCI [12-13]. Kurtul et al. showed similar results but included patients with all types of ACS; however, more than $60 \%$ of them were STEMI patients [14]. The predictive utility for RDW is most likely explained by the fact that it signifies ongoing systemic inflammation [15]. Meanwhile, elevated RDW levels are 
consistent with increased anisocytosis among red blood cells, likely due to impaired erythrocyte maturation. Subsequently, this might indirectly reflect a state of systemic inflammation and constant exposure to oxidative stress and thus increased CI-AKI risk. However, in our analysis, this was not the case. This is most likely explained by the fact that we included patients with STEMI and NSTEMI as we believe CI-AKI prediction is more essential in patients with NSTEMI as there is some room for precautious measures prior to receiving contrast. Moreover, and since NSTEMI patients usually have more comorbidities at baseline than STEMI patients, we believe that other baseline characteristics are more important for CI-AKI prediction.

In summary, although RDW is a marker of inflammation that has a negative prognostic value in $\mathrm{CAD}$ and is linked to kidney dysfunction, the role of the usual predictive markers, such as heart failure and glomerular filtration rate (GFR), is more important. In our study, there was no significant difference in values between the two groups, and RDW didn't provide an additional benefit to MRS. Therefore, we conclude that MRS remains the most useful predictive marker for CI-AKI.

\section{Conclusions}

RDW does not correlate with the development of CI-AKI in patients with ACS. Mehran risk score remains a better indicator for CI-AKI risk assessment with no role for the addition of RDW to it. Further studies are needed to better assess predictors of CI-AKI in patients undergoing percutaneous coronary intervention.

\section{Additional Information \\ Disclosures}

Human subjects: All authors have confirmed that this study did not involve human participants or tissue. Animal subjects: All authors have confirmed that this study did not involve animal subjects or tissue. Conflicts of interest: In compliance with the ICMJE uniform disclosure form, all authors declare the following: Payment/services info: All authors have declared that no financial support was received from any organization for the submitted work. Financial relationships: All authors have declared that they have no financial relationships at present or within the previous three years with any organizations that might have an interest in the submitted work. Other relationships: All authors have declared that there are no other relationships or activities that could appear to have influenced the submitted work.

\section{References}

1. Mamoulakis C, Tsarouhas K, Fragkiadoulaki I, et al: Contrast-induced nephropathy: basic concepts, pathophysiological implications and prevention strategies. Pharmacol Ther. 2017, 180:99-112. 10.1016/j.pharmthera.2017.06.009

2. McCullough PA: Contrast-induced nephropathy. Interv Cardiol Clin. 2014, 3:357-362. 10.1016/j.iccl.2014.03.002

3. Atanda AC, Olafiranye O: Contrast-induced acute kidney injury in interventional cardiology: emerging evidence and unifying mechanisms of protection by remote ischemic conditioning. Cardiovasc Revasc Med. 2017, 18:549-553. 10.1016/j.carrev.2017.06.001

4. Senoo T, Motohiro M, Kamihata H, et al.: Contrast-induced nephropathy in patients undergoing emergency percutaneous coronary intervention for acute coronary syndrome. Am J Cardiol. 2010, 105:624-628. 10.1016/j.amjcard.2009.10.044

5. McCullough PA: Contrast-induced acute kidney injury. J Am Coll Cardiol. 2008, 51:1419-1428. 10.1016/j.jacc.2007.12.035

6. Mehran R, Aymong ED, Nikolsky E, et al.: A simple risk score for prediction of contrastinduced nephropathy after percutaneous coronary intervention: development and initial validation. J Am Coll Cardiol. 2004, 44:1393-1399. 10.1016/j.jacc.2004.06.068

7. Silver SA, Shah PM, Chertow GM, Harel S, Wald R, Harel Z: Risk prediction models for 
contrast induced nephropathy: systematic review. BMJ. 2015, 351:4395. 10.1136/bmj.h4395

8. Waikar SS, Liu KD, Chertow GM: Diagnosis epidemiology and outcomes of acute kidney injury. Clin J Am Soc Nephrol. 2008, 3:844-861. 10.2215/CJN.05191107

9. Danese E, Lippi G, Montagnana M: Red blood cell distribution width and cardiovascular diseases. J Thorac Dis. 2015, 7:402. 10.3978/j.issn.2072-1439.2015.10.04

10. Bozorgi A, Khaki S, Mortazavi SH, Sadeghian S, Khoshnevis M, Tofighi S, Khaki A: Effect of baseline red blood bell distribution width on short-and intermediate-term mortality of patients under primary percutaneous coronary intervention: a survival analysis. Crit Pathw Cardiol. 2016, 15:69-74. 10.1097/HPC.0000000000000063

11. Mizuno A, Ohde S, Nishizaki Y, Komatsu Y, Niwa K: Additional value of the red blood cell distribution width to the Mehran risk score for predicting contrast-induced acute kidney injury in patients with ST-elevation acute myocardial infarction. J Cardiol. 2015, 66:41-45. 10.1016/j.jjcc.2014.09.006

12. Akin F, Celik O, Altun I, et al.: Relation of red cell distribution width to contrast-induced acute kidney injury in patients undergoing a primary percutaneous coronary intervention. Coron Artery Dis. 2015, 26:289-295. 10.1097/MCA.0000000000000223

13. Akkoyun DC, Akyüz A, Kurt Ö, Bilir B, Alpsoy S, Güler N: Relationship between red cell distribution width and contrast-induced nephropathy in patients who underwent primary percutaneous coronary intervention. Turk Kardiyol Dern Ars. 2015, 43:613-620. 10.5543/tkda.2015.37941

14. Kurtul A, Yarlioglues M, Murat SN, et al.: Red cell distribution width predicts contrastinduced nephropathy in patients undergoing percutaneous coronary intervention for acute coronary syndrome. Angiology. 2015, 66:433-440. 10.1177/0003319714535238

15. Bujak K, Wasilewski J, Osadnik T, et al.: The prognostic role of red blood cell distribution width in coronary artery disease: a review of the pathophysiology. Dis Markers. 2015, 2015:824624. 10.1155/2015/824624 